\title{
Sum-Rule Inequalities and a Toy Model Paradox
}

\author{
T.G. Steele, S. Alavian, J. Kwan \\ Department of Physics $\&$ Engineering Physics and \\ Linear Accelerator Laboratory \\ University of Saskatchewan \\ Saskatoon, Saskatchewan S7N 0W0, Canada
}

(August 28, 1996)

\begin{abstract}
Fundamental inequalities for QCD sum-rules are applied to resolve a paradox recently encountered in a sum-rule calculation [1]. This paradox was encountered in a toy model known to be free of resonances that yields an apparent resonance using a standard sum-rule stability analysis. Application of the inequalities does not support the existence of a well defined sum-rule calculation, and shows a strong distinction from typical behaviour in QCD.
\end{abstract}

QCD sum-rules [2 [5] have demonstrated their utility in numerous theoretical determinations of hadronic properties. In this approach the QCD condensates parametrize nonperturbative aspects of the vacuum, and are an essential feature of the sum-rules used to determine hadronic properties.

An apparent paradox in a sum-rule calculation has recently been encountered [1]. This paradox consists of a toy model known to be free of resonances that yields an apparent resonance after the standard sum-rule stability method is applied to the truncated correlation function. Clearly this raises concerns about the reliability of the stability criteria used to obtain predictions from QCD sum-rules.

Fundamental inequalities for sum-rules have recently been developed [6]. These inequalities must be satisfied if the sum-rule is consistent with an integrated cross-section, but the analysis does not require detailed phenomenological input on the nature of this crosssection. These inequalities thus provide novel, valuable criteria for studying the validity and self-consistency of a sum-rule calculation. QCD sum-rule inequalities are also of phenomenological value since they have been utilized to obtain bounds related to the electromagnetic polarizability of charged pions [7].

Laplace or Borel sum-rules are derived from a typical subtracted dispersion relation

$$
-\frac{d \Pi\left(Q^{2}\right)}{d Q^{2}}=\frac{1}{\pi} \int_{t_{0}}^{\infty} \frac{\operatorname{Im} \Pi(t)}{\left(t+Q^{2}\right)^{2}} d t
$$

where $t_{0}$ is a physical threshold. The Borel transform operator $\hat{B}[2]$ is then applied to the dispersion relation and the continuum is subtracted leading to the Laplace sum-rules.

$$
\mathcal{R}_{k}\left(\tau, s_{0}\right)=\frac{1}{\tau} \hat{B}\left[Q^{2} k \Pi\left(Q^{2}\right)\right]-\text { continuum }=\frac{1}{\pi} \int_{t_{0}}^{s_{0}} t^{k} e^{-t \tau} \operatorname{Im} \pi(t) d t
$$


The parameter $s_{0}$ represents the continuum threshold leading to duality between the field theory and the physical process related to $\operatorname{Im} \Pi(t)$.

Hölder's inequality [8,9] for integrals defined over a measure $d \mu$ is

$$
\begin{aligned}
\left|\int_{t_{1}}^{t_{2}} f(t) g(t) d \mu\right| & \leq\left(\int_{t_{1}}^{t_{2}}|f(t)|^{p} d \mu\right)^{1 / p}\left(\int_{t_{1}}^{t_{2}}|g(t)|^{q} d \mu\right)^{1 / q}, \\
\frac{1}{p}+\frac{1}{q} & =1 ; \quad p, q \geq 1 .
\end{aligned}
$$

When $p=q=2$ the Hölder inequality reduces to the well known Schwarz inequality. The key idea in applying Hölder's inequality to sum-rules is recognizing that for a typical correlation function $\Pi\left(Q^{2}\right)$, Im $\Pi(t)$ is positive because of its relation to physical cross-sections and can thus serve as the measure $d \mu=\operatorname{Im} \Pi(t) d t$ in (3). Returning to (3) with $d \mu=\operatorname{Im} \Pi(t) d t$, $f(t)=t^{\alpha} e^{-a t \tau}, g(t)=t^{\beta} e^{-b t \tau}$ and appropriate integration limits we find

$$
\mathcal{R}_{\alpha+\beta}\left(\tau, s_{0}\right) \leq \mathcal{R}_{\alpha p}^{1 / p}\left(a p \tau, s_{0}\right) \mathcal{R}_{\beta q}^{1 / q}\left(b q \tau, s_{0}\right) ; \quad a+b=1 \quad .
$$

Imposing restrictions that we have the integer values $k$ needed for the sum-rules (2) leads to the following set of inequalities.

$$
\begin{aligned}
& \mathcal{R}_{0}\left[\omega \tau_{\text {min }}+(1-w) \tau_{\text {max }}, s_{0}\right] \leq \mathcal{R}_{0}^{\omega}\left[\tau_{\text {min }}, s_{0}\right] \mathcal{R}_{0}^{1-\omega}\left[\tau_{\text {max }}, s_{0}\right] \\
& \mathcal{R}_{1}\left[\omega \tau_{\text {min }}+(1-w) \tau_{\text {max }}, s_{0}\right] \leq \mathcal{R}_{1}^{\omega}\left[\tau_{\text {min }}, s_{0}\right] \mathcal{R}_{1}^{1-\omega}\left[\tau_{\text {max }}, s_{0}\right] \\
& \mathcal{R}_{1}\left[\frac{\tau_{\text {min }}+\tau_{\text {max }}}{2}, s_{0}\right] \leq \mathcal{R}_{2}^{1 / 2}\left[\tau_{\text {min }}, s_{0}\right] \mathcal{R}_{0}^{1 / 2}\left[\tau_{\text {max }}, s_{0}\right], \\
& \mathcal{R}_{1}\left[\frac{\tau_{\text {min }}+\tau_{\text {max }}}{2}, s_{0}\right] \leq \mathcal{R}_{0}^{1 / 2}\left[\tau_{\text {min }}, s_{0}\right] \mathcal{R}_{2}^{1 / 2}\left[\tau_{\text {max }}, s_{0}\right] \\
& 0 \leq \omega \leq 1 \quad ; \quad \tau_{\text {min }} \leq \tau_{\text {max }}
\end{aligned}
$$

Similar inequalities can be obtained for higher sum-rules with $k \geq 2$. Furthermore, for small $\delta \tau \equiv \tau_{\max }-\tau_{\min }(7)$ and (8) are in principle contained in the first two inequalities.

In summary, if the sum-rules are a valid and consistent representation of the integration of $\operatorname{Im} \Pi(t)$ in (2) then the sum-rules $\mathcal{R}_{k}\left(\tau, s_{0}\right)$ must satisfy the following fundamental inequalities.

$$
\begin{aligned}
\rho_{0} \equiv \frac{\mathcal{R}_{0}\left[\tau+(1-\omega) \delta \tau, s_{0}\right]}{\mathcal{R}_{0}^{\omega}\left[\tau, s_{0}\right] \mathcal{R}_{0}^{1-\omega}\left[\tau+\delta \tau, s_{0}\right]} \leq 1 & \forall 0 \leq \omega \leq 1 \\
\rho_{1} \equiv \frac{\mathcal{R}_{1}\left[\omega \tau+(1-\omega) \delta \tau, s_{0}\right]}{\mathcal{R}_{1}^{\omega}\left[\tau, s_{0}\right] \mathcal{R}_{1}^{1-\omega}\left[\tau+\delta \tau, s_{0}\right]} \leq 1 & \forall 0 \leq \omega \leq 1
\end{aligned}
$$

Provided that $\delta \tau$ is reasonably small (in $\mathrm{QCD} \delta \tau \approx 0.1 \mathrm{GeV}^{-2}$ appears sufficient) these inequalities are insensitive to the value of $\delta \tau$, permitting a simple determination of the $\tau, s_{0}$ parameter space satisfying the inequalities.

In the standard stability analysis of QCD sum-rules, the ratio $\mathcal{R}_{1} / \mathcal{R}_{0}$ gives the mass of the lightest resonance in the simple "resonance plus continuum" model for $\operatorname{Im} \Pi(t)$. Thus if this stability analysis is to be consistent with Hölder's inequalities then (10) and (11) should be satisfied in the same region of $\tau, s_{0}$ parameter space in which the stability analysis occurs. The $s_{0}, \tau$ parameter space consistent with the inequalities (10,11) is shown in Figures 1-3 for a wide variety of QCD sum-rule applications ranging from light to heavy quark systems 
22 5.10] Also shown in the figures is the the range of $\tau, s_{0}$ resulting from the corresponding stability analysis [2 20,10]. In all cases, a large portion of the stability region is seen to be consistent with the inequalities, indicating a reliable sum-rule analysis. The effect of uncertainties intrinsic to the sum-rule method has been analyzed in [6], and the result is a slight increase in the area of the parameter space consistent with the inequalities.

The allowed sum-rule parameter space in Figures 1-3 exhibits two crucial features that we believe are characteristic of QCD: a failure of duality below a critical value of $s_{0}$, and an upper limit on $\tau$ corresponding to a lower bound on the energy. It is also interesting to note that the narrow-width single resonance approximation provides a phenomenological contribution on the right-hand side of (2) given by $F^{2} e^{-M^{2} \tau}$. This satisfies the equality in $(10,11)$, and so the borders of the parameter space in Figures 1-3 represents points where the sum-rule is in good agreement with a single (narrow-width) resonance model. This provides a practical criteria for choosing the optimal $s_{0}$ in a sum-rule analysis: a value of $s_{0}$ which corresponds to a wide, flat border of the allowed parameter space has maximum agreement with a single narrow-width resonance. As is seen from consideration of the values of $s_{0}$ used in the actual sum-rule analyses (horizontal lines in Figures 1-3) [2 5.10] this criteria is consistent with the range of $s_{0}$ corresponding to the conventional stability analysis.

We now consider the application of the inequalities to the toy model of [1] where a correlator of composite operators is calculated in the ladder approximation for a $\phi^{3}$ theory. The spectral density in this model has a non-physical behaviour in some momentum regions where the ladder diagrams do not accurately represent the exact correlation function. The following sum-rules can be obtained from a truncated version of the correlator: ?

$$
\begin{aligned}
& \mathcal{R}_{0}\left(\tau, s_{0}\right)=\frac{1-e^{-s_{0} \tau}}{\tau}-6 \zeta(3)+20 \zeta(5) \tau-35 \zeta(7) \tau^{2}+42 \zeta(9) \tau^{3}+\ldots \\
& \mathcal{R}_{1}\left(\tau, s_{0}\right)=\frac{1-\left(1+s_{0} \tau\right) e^{-s_{0} \tau}}{\tau^{2}}-20 \zeta(5)+70 \zeta(7) \tau-129 \zeta(9) \tau^{2}+\ldots
\end{aligned}
$$

Despite the non-physical aspects of this model, when the truncated correlator is analysed with conventional sum-rule stability techniques, an apparently physical resonance and continuum of positive spectral density is discovered in contradiction with the non-truncated ladder approximation [1]. This clearly raises concerns about the reliability of conventional QCD sum-rule stability techniques where information on physical resonances is extracted from truncated versions of QCD correlation functions.

The $s_{0}, \tau$ parameter space where the toy model (12,13) is consistent with the inequalities $(10,11)$ is shown in Figure 4. Also shown is a boxed region corresponding to the range of $\tau$ and $s_{0}$ obtained from the stability analysis [1] (an uncertainty for $s_{0}$ was not given in [1], so a $10 \%$ deviation has been assumed for the quoted optimal value of $s_{0}=16.6$ at the lower bound of the boxed region). In contrast to the QCD examples, there is no overlap between the stability and inequality parameter space, indicating an inconsistency with the fundamental inequalities. The parameter space consistent with the inequalities bears little resemblance to the $\mathrm{QCD}$ examples since the flat border of the parameter space needed for maximal agreement with a single narrow resonance is absent.

\footnotetext{
${ }^{1}$ The notation $\tau=1 / M^{2}$ is used in [1].
} 
Thus in contrast with the conventional sum-rule stability techniques which identify a spurious resonance and physical continuum in the toy model, application of fundamental sum-rule inequalities reveals that this toy model does not provide a convincing description of a resonance and physical continuum. Furthermore, the inequalities provide a clear distinction between the toy model and QCD. This indicates the importance of supplementing the traditional sum-rule stability analysis with the fundamental inequalities developed in [6].

Acknowledgements: TGS and JK are grateful for the financial support of the Natural Sciences and Engineering Research Council of Canada (NSERC). We are grateful to M. Lavelle for bringing reference [1] to our attention. TGS dedicates this work to the memory of G. Fitzhenry. 


\section{REFERENCES}

[1] A.A. Penin, A.A. Pivovarov, Phys. Lett. 357B, 427 (1995).

[2] M.A. Shifman, A.I. Vainshtein, V.I. Zakharov, Nucl. Phys. B147, 385 (1979).

[3] M.A. Shifman, A.I. Vainshtein, V.I. Zakharov, Nucl. Phys. B147, 448 (1979).

[4] L.J. Reinders, H. Rubenstein, S. Yazaki, Phys. Rep. 127, 1 (1985).

[5] S. Narison, QCD Spectral Sum-Rules, Lecture Notes in Physics v. 26 (World Scientific, Singapore, 1989).

[6] M. Benmerrouche, G. Orlandini, T.G. Steele, Phys. Lett. 356B 573, (1995).

[7] M. Benmerrouche, G. Orlandini, T.G. Steele, Phys. Lett. 366B 354, (1996)

[8] E.F. Beckenbach, R. Bellman, Inequalities, (Springer, Berlin, 1961).

[9] S.K. Berberian, Measure and Integration, (MacMillan, New York, 1965).

[10] E. Bagan, P. Ball, V.M. Braun, H.G. Dosch, Phys. Lett. 278B, 457 (1992).

[11] D. Leinweber, "QCD Sum Rules for Skeptics", nucl-th/9510051 


\section{FIGURES}

FIG. 1. The shaded area represents the region in the $s_{0}, \tau$ parameter space consistent with the inequalities for the light-quark vector sum-rule related to the $\rho$ meson [2 5]. The boxed region indicates the values of $\tau, s_{0}$ used in the sum-rule stability analysis. As determined in [11], a 25\% uncertainty in $s_{0}$ has been used.

FIG. 2. Same as in Figure 1 except for the light quark axial-vector sum-rule related to the $A_{1}$ meson [2].

FIG. 3. Same as Figure 1 except for the heavy quark sum-rule related to the $B$ meson [10]. The convention for $s_{0}$ and $\tau$ differs from Figures 1 and 2 for consistency with [10] which takes into

account the relation with heavy quark effective theories. In particular $\tau=1 / \Delta M$ in the notation of [10].

FIG. 4. Shaded region indicates the parameter space consistent with the inequalities for the sum-rule in the toy model [1]. The value $\delta \tau \approx 0.001$ has chosen because of the lower scales for $\tau$ relevant to the toy model. The boxed region indicates the values of $\tau, s_{0}$ used in the sum-rule stability analysis [四]. 
This figure "fig1-1.png" is available in "png" format from: http://arxiv.org/ps/hep-ph/9701267v1 
This figure "fig1-2.png" is available in "png" format from: http://arxiv.org/ps/hep-ph/9701267v1 
This figure "fig1-3.png" is available in "png" format from: http://arxiv.org/ps/hep-ph/9701267v1 
This figure "fig1-4.png" is available in "png" format from: http://arxiv.org/ps/hep-ph/9701267v1 